\title{
Nuclear moments put a new spin on the structure of 131 In
}

\section{Adam Vernon ( $\nabla$ vernona@mit.edu )}

Massachusetts Institute of Technology https://orcid.org/0000-0001-8130-0109

\section{Ronald Fernando Garcia Ruiz}

Massachusetts Institute of Technology https://orcid.org/0000-0002-2926-5569

\section{T. Miyagi}

TRIUMF, 4004 Wesbrook Mall, Vancouver, BC V6T 2A3, Canada

\section{Cory Binnersley}

The University of Manchester

\section{Jon Billowes}

The University of Manchester

\section{Mark Bissell}

The University of Manchester

Jeremy Bonnard

University of York https://orcid.org/0000-0002-5164-3343

\section{Thomas Cocolios}

KU Leuven

\section{Jacek Dobaczewski}

University of York https://orcid.org/0000-0002-4158-3770

\section{Kieran Flanagan}

The University of Manchester

\section{George Georgiev}

Universite Paris-Saclay

\section{Wouter Gins}

University of Jyväskylä

\section{Ruben de Groote}

University of Jyväskylä

\section{Reinhart Heinke}

CERN

\section{Jason Holt}

TRIUMF https://orcid.org/0000-0003-4833-7959

Ágota Koszorús

University of Liverpool

David Leimbach 
CERN https://orcid.org/0000-0002-4587-1067

\section{Kara Lynch}

CERN

\section{Gerda Neyens}

KU Leuven

\section{Steven Stroberg}

University of Washington https://orcid.org/0000-0002-0635-776X

\section{Shane Wilkins}

European Organization for Nuclear Research https://orcid.org/0000-0001-8897-7227

\section{Xiaofei Yang}

Peking University https://orcid.org/0000-0002-1633-4000

\section{Deyan Yordanov}

CERN

\section{Gregory Farooq-Smith}

KU Leuven https://orcid.org/0000-0001-8384-7626

\section{Jeroen Hustings}

KU Leuven

\section{Physical Sciences - Article}

Keywords: nuclear physics, electromagnetism, 131ln

Posted Date: June 17th, 2021

DOI: https://doi.org/10.21203/rs.3.rs-611360/v1

License: (c) (i) This work is licensed under a Creative Commons Attribution 4.0 International License. Read Full License

Version of Record: A version of this preprint was published at Nature on July 13th, 2022. See the published version at https://doi.org/10.1038/s41586-022-04818-7. 


\title{
Nuclear moments put a new spin on the structure of ${ }^{131}$ In
}

\author{
A.R. Vernon ${ }^{1,2, *}$, R.F. Garcia Ruiz ${ }^{2,3, *}$, T. Miyagi ${ }^{4}$, C.L. Binnersley ${ }^{1}$, J. Billowes ${ }^{1}$, \\ M.L. Bissell ${ }^{1}$, J. Bonnard ${ }^{5}$, T.E. Cocolios ${ }^{6}$, J. Dobaczewski ${ }^{5,7}$, G.J. Farooq-Smith ${ }^{6}$, \\ K.T. Flanagan ${ }^{1,8}$, G. Georgiev ${ }^{9}$, W. Gins ${ }^{6,10}$, R.P. de Groote ${ }^{6,10}$, R. Heinke ${ }^{3}$, J.D. Holt ${ }^{4,11}$, \\ J. Hustings ${ }^{6}$, Á. Koszorús ${ }^{6}$, D. Leimbach ${ }^{12,13,14}$, K.M. Lynch ${ }^{3}$, G. Neyens ${ }^{6,3}$, \\ S.R. Stroberg ${ }^{15}$, S.G. Wilkins ${ }^{1,2}$, X.F. Yang ${ }^{6,16}$, and D.T. Yordanov ${ }^{3,9}$
}

\author{
${ }^{1}$ School of Physics and Astronomy, The University of Manchester, Manchester M13 9PL, United Kingdom \\ ${ }^{2}$ Massachusetts Institute of Technology, Cambridge, MA 02139, USA \\ ${ }^{3}$ EP Department, CERN, CH-1211 Geneva 23, Switzerland \\ ${ }^{4}$ TRIUMF,4004 Wesbrook Mall, Vancouver, BC V6T 2A3, Canada \\ ${ }^{5}$ Department of Physics, University of York, Heslington, York YO10 5DD, United Kingdom \\ ${ }^{6} \mathrm{KU}$ Leuven, Instituut voor Kern- en Stralingsfysica, B-3001 Leuven, Belgium \\ ${ }^{7}$ Institute of Theoretical Physics, Faculty of Physics, University of Warsaw, ul. Pasteura 5, PL-02-093 Warsaw, \\ Poland \\ ${ }^{8}$ Photon Science Institute, The University of Manchester, Manchester M13 9PL, UK \\ ${ }^{9}$ IJCLab, CNRS/IN2P3, Université Paris-Saclay, 91405 Orsay, France \\ ${ }^{10}$ Department of Physics, University of Jyväskylä, PB 35(YFL) FIN-40351 Jyväskylä, Finland \\ ${ }^{11}$ Department of Physics, McGill University, 3600 Rue University, Montréal, QC H3A 2T8, Canada \\ ${ }^{12}$ EN Department, CERN, CH-1211 Geneva 23, Switzerland \\ ${ }^{13}$ Institut für Physik, Johannes Gutenberg-Universität Mainz, D-55128 Mainz, Germany \\ ${ }^{14}$ Department of Physics, The University of Gothenburg, Gothenburg, Sweden \\ ${ }^{15}$ Department of Physics, University of Washington, Seattle, Washington 98105, USA \\ ${ }^{16}$ School of Physics and State Key Laboratory of Nuclear Physics and Technology, Peking University, Beijing \\ 100871, China \\ *Corresponding authors: vernona@mit.edu, rgarciar@mit.edu
}

In spite of the high-density and strongly correlated nature of the atomic nucleus, experimental and theoretical evidence suggests that around particular 'magic' numbers of nucleons, nuclear properties are governed by a single unpaired nucleon ${ }^{1,2}$. A microscopic understanding of the extent of this behavior and its evolution in neutron-rich nuclei remains an open question in nuclear physics ${ }^{3-5}$. A textbook example is the electromagnetic moments of indium $(Z=49)^{6}$, which are dominated by a hole with respect to the proton magic number $Z=50$ nucleus. They exhibit a remarkably constant behavior over a large range of odd-mass isotopes, previously interpreted as pure "single-particle behaviour". Here, we present precision laser spectroscopy measurements performed to investigate the validity of this simple single-particle picture. Observation of an abrupt change in the dipole moment at $N=82$ reveals that while the single-particle picture indeed dominates at neutron magic number $N=82^{2,7}$, it does not for previously studied isotopes. We present state-of-the-art nuclear theory developed to investigate the details of the nuclear forces that describe the experimental results. The emergence and disappearance of single-particle behavior was reproduced from an ab initio theory, including challenging many-body correlations in these large, complex nuclei. The inclusion of time-symmetry-breaking mean fields is shown to be essential for a correct description of the nuclear electromagnetic properties within the Density Functional Theory framework. Until now, such time-odd channels have been poorly constrained, but are essential to provide accurate predictions of nuclear properties necessary for searches of new physics with neutrinos ${ }^{8,9}$ and studies of fundamental symmetries ${ }^{10,11}$. These findings are key to understand the microscopic origin of nuclear electromagnetism and the emergence of single-particle phenomena from complex nuclei.

\section{Introduction}

The atomic nucleus is formed by strongly-interacting nucleons (protons, $Z$, and neutrons, $N$ ), packed tightly into a volume around a trillion times smaller than that of atoms. These highdensity systems are bound by the strong nuclear force which, in contrast to the well understood electromagnetic force that dominates the properties of atoms and molecules, is exceedingly more complex and not yet fully understood. Hence, describing the atomic nuclei and predicting their properties at extreme values of mass and charge are major long-standing challenges for nuclear science.
Similar to electrons in an atom, the nucleons (protons and neutrons) in the atomic nucleus occupy quantum 'shells'. Thus, nuclei with a single valence particle or hole around a nuclear closed shell provide important foundations for our understanding of the atomic nucleus. Their simpler structure vastly reduces the complexity of the quantum many-body problem, providing critical guidance for the development of nuclear theory.

Recent advances in our understanding of the strong interaction and the development of many-body methods, combined with escalation in computer power, have enabled theoretical 

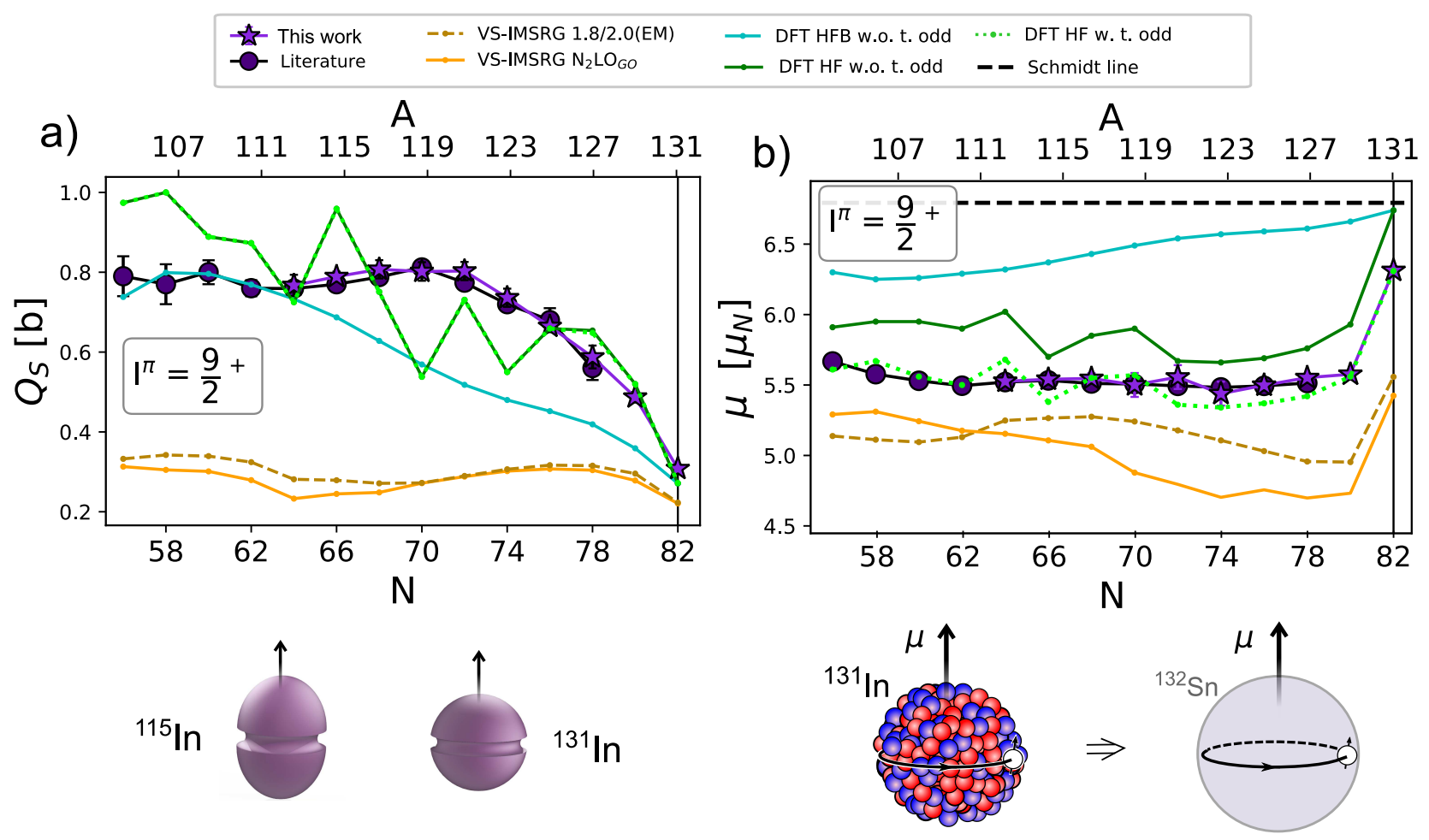

Figure 1. (color online) Evolution of nuclear electromagnetic properties for the $9 / 2^{+}$ground states of ${ }^{105-131}$ In isotopes: a) electric quadrupole moments; and b) magnetic dipole moments. The horizontal dotted line indicates the single-particle value (Schmidt line). Experimental results are compared with theoretical calculations from ab initio (VS-IMSRG) and Density Functional Theory (DFT). Literature experimental values for ${ }^{105-127}$ In were taken from Ref. ${ }^{12}$. The evolution of single-particle properties of these isotopes are illustrated at the bottom of the figure. At neutron magic number $N=82$, the electromagnetic properties approach the pure single-particle value (see text for more details).

descriptions of increasingly complex nuclei. Isotopes around the proton closed shell $Z=50$, are now the frontier of $a b$ initio calculations ${ }^{13,14}$. The properties of these nuclei can be described by complementary many-body methods such as configuration-interaction methods ${ }^{4}$ and nuclear Density Functional Theory (DFT $)^{15}$. This has lead to an increased focus on studying this region of the nuclear chart (around $Z=50$, $N=50,82$ ) over the last decade $2,7,16-18$.

Here we present measurements of two fundamental properties of indium isotopes using precision laser spectroscopy: the (spectroscopic) magnetic dipole moment, $\mu$, and electric quadrupole moment, $Q$. Measurements were performed for the neutron-rich In $(Z=49)$ isotopes, reaching up to ${ }^{131} \mathrm{In}$, which possesses a magic number of $N=82$ neutrons (see Methods section for details). With a proton hole in the closedshell of $Z=50$, the low-energy structure of the odd-mass In isotopes is expected to be governed by a single-hole configuration in the proton orbit $\pi 1 g_{9 / 2}$.

As we show here, the nuclear magnetic dipole moments of odd-mass In isotopes are determined by the total spin distribution of the nucleus induced by the unpaired valence proton hole. This is schematically illustrated at the bottom right of
Figure 1. The nuclear electric quadrupole moment provides a complementary measurement of the nuclear charge distribution, and is highly sensitive to the collective motion of all nucleons ${ }^{19}$. These observables together therefore probe distinct aspects of the nucleon distribution and measuring them across a large range of neutron numbers allows a unique insight into the evolution of the interplay between single-particle and collective nuclear phenomena.

Previously, the magnetic and quadrupole moments of the ground state $I^{\pi}=9 / 2^{-}$of indium isotopes were known to exhibit remarkably little variation over 22 isotopes, from $A=105$ to 127 (see Figure $1 \mathrm{~b}$ ) $)^{12}$. The constant value of the magnetic moment over such long range of isotopes has been presented as an archetypal example of the independentparticle behaviour of single-particle states near a proton shell closure $^{6,12}$. 'How do these seemingly simple patterns emerge from complex interactions among protons and neutrons?' and 'Do they prevail at extreme number of neutrons?' are two major open questions that we address in this work.

In addition to the ground state, the indium isotopes can also exist in excited nuclear configurations with relatively long lifetimes - isomers - with spin $I^{\pi}=1 / 2^{-}$. These isomeric states 
provide additional insight and are expected to be described by a single-hole configuration based on a different proton orbital $\left(\pi 2 p_{1 / 2}\right)$. However, in contrast to the $I^{\pi}=9 / 2^{+}$states, the $\mu$ values of these isomeric states exhibit significant variations, posing a three-decades-long puzzle in our description of these nuclei ${ }^{12}$.

To unravel the microscopic origin of the electromagnetic properties of these isotopes, we compare our experimental results with two complementary state-of-the-art theoretical methods: i. ab initio valence-space in-medium similarity renormalisation group (VS-IMSRG) calculations ${ }^{13,20}$, which start from nucleon-nucleon interactions derived from chiral effective field theory, constrained by the properties of up to only four nucleon systems ${ }^{21,22}$; and ii. symmetry-breaking nuclear Density Functional Theory (DFT) ${ }^{15,23}$. The latter assumes nucleons moving within their own self-consistently-generated spin-dependent broken-symmetry-confining potential. DFT provides a satisfactory description of bulk nuclear properties such as radii and binding energies across the whole nuclear chart $^{24-26}$. Here, we have developed its symmetry-restored version $^{27}$ to be able to provide accurate calculations of spectroscopic $\mu$ and $Q$ moments.

\section{Experimental and Theoretical developments}

Our measurements were performed using the collinear resonance ionisation spectroscopy (CRIS) technique at the ISOLDE facility of CERN ${ }^{28}$ (see Methods section for details). From the hyperfine structure, we extracted the magnetic dipole and electric quadrupole parameters, $A_{h f}, B_{h f}$ of the probed atomic states, corresponding to the two long-lived nuclear states, $9 / 2^{+}$and $1 / 2^{-}$, present in each isotope. Recent improvements in the sensitivity of the technique allowed us to achieve high-resolution spectroscopy measurements, despite production of the indium isotopes at rates below 1000 atoms/s in the presence of large isobaric contamination. Prior to the study of these exotic isotopes, sensitive ionisation schemes and atomic theory calculations had to be developed to accurately extract the nuclear properties from spectroscopy measurements ${ }^{29,30}$.

We performed ab initio VS-IMSRG calculations (see Methods for further details) using two different sets of initial two$(\mathrm{NN})$ and three-nucleon $(3 \mathrm{~N})$ forces derived from chiral effective field theory ${ }^{31,32}$; the $1.8 / 2.0(\mathrm{EM})^{21,22}$ and the more recent $\mathrm{N}^{2} \mathrm{LO}_{\mathrm{GO}}{ }^{33}$. The $1.8 / 2.0(\mathrm{EM})$ set is constrained only by fitting to properties of two-, three-, and four-nucleon systems. $\mathrm{N}^{2} \mathrm{LO}_{\mathrm{GO}}$ was recently developed to include $\Delta$-isobar degrees of freedom and is additionally fit to reproduce saturation properties of infinite nuclear matter ${ }^{33}$.

We performed DFT calculations using both Hartree-Fock (HF) or Hartree-fock-Bogilibov (HFB) approaches, corresponding to configurations of the nucleus constructed using single-nucleon (HF), or nucleon-hole pair excitations (HFB) as basis states, with HFB calculations introducing pairing correlations. The electromagnetic moments of semi-magic \pm 1 nucleon systems, such as the indium isotopes, are well suited to study DFT time-symmetry-breaking (time-odd) contributions to the mean field ${ }^{34,35}$, which vary with the time-reversal operator. These fields are predominantly generated by the two-body spin-spin interaction terms, and up until now were poorly constrained within DFT theory. However, they are of particular interest as our understanding of time-reversalviolating mean fields is critical to tackle open problems of modern physics, e.g. the search for new physics ${ }^{8-11}$ and dark matter searches ${ }^{36}$. Our experimental results presented an excellent opportunity to perform and test these developments. To investigate the relative importance of time-odd fields and pairing correlations, DFT calculations were performed by turning "on" and "off" of each effect. The results of both $a b$ initio and DFT types of calculation are shown alongside the experimental $Q$ and $\mu$ moments in Figure 1a) and 1b), respectively, for the $9 / 2^{+}$states of ${ }^{105-131}$ In. The $\mu$ moments of the $1 / 2^{-}$states are shown in Figure 2. All experimental data are presented in Tables 1 and 2 of Methods, and compared with literature values that exist for ${ }^{105-127} \mathrm{In}$.

\section{Results and discussion}

In the single-particle picture, a proton hole induces an intrinsic prolate deformation of the whole nucleus, as indicated schematically in Figure 1 (bottom left). A gradual decrease in the $Q$ values of the $9 / 2^{+}$states was previously observed up to $N=78$. Our measurements reveal a notably larger decrease at $N=82$, indicating a significant decrease in deformation (Figure 1a). The VS-IMSRG calculations reproduce the experimental trends, i.e local variations in neutron number, a dip around $N=64$, and a gradual decrease towards $N=82$. However, the magnitude of the $Q$ is underestimated. The reproduction of the magnitude of the quadrupole moments is a known challenge for $a b$ initio nuclear theory, as the $Q$ moments are a highly collective emergent property of the nucleus which can require the inclusion of extensive many-body correlations ${ }^{37}$. Traditionally, phenomenological calculations are fitted to match the experimental data by assigning empirical 'effective' charges to valence neutrons ${ }^{38}$. Neither effective charges nor effective $g$-factors were used in our $a b$ initio or DFT calculations. This work therefore provides an important insight into how a correct description of these nuclei and single-particle behaviour emerges intrinsically from our calculations without the use of commonly used effective factors.

Conversely, our DFT calculations are able to closely reproduce the overall magnitude of the $Q$ moments. As $N=82$ is approached, the agreement with the calculations without pairing (HF), shows that describing individual neutron orbitals becomes important. However, due to effects induced by occupying individual neutron orbitals, an inaccurate staggering with neutron number is also produced, compared to HFB. As shown in Figure 1a), time-odd contributions have a negligible effect on $Q$ moments.

In contrast to the $Q$ moments, the $\mu$ moments of the $9 / 2^{+}$ states were known to exhibit little variation ${ }^{12}$, which is continued up to ${ }^{129}$ In in our observation. However, we observe 
an abrupt increase at ${ }^{131} \mathrm{In}$, see Figure $1 \mathrm{~b}$ ), and the atomic spectra in Figure 3. The extreme single-particle magnetic moment of a proton in the $\pi g_{9 / 2}$ orbit, the so-called 'Schmidt' limit $^{39}$, is also indicated in Figure 1b). Deviations from the extreme single-particle value have usually been attributed to a mean effect of the other nucleons, and are typically taken into account using an empirical 'quenched' or 'effective' $g$ factor $^{12}$. Our results now reveal a value much closer to the extreme single-particle limit at $N=82$, indicating the $\mu$ moments for $N<82$ in fact depart from the single-particle picture. This illustrates how the use of 'effective' operators can lead to contradictory conclusions and obscure critical details and changes in the underlying nuclear structure. In contrast to the quadrupole moments, we find that the inclusion of time-odd fields proves to be essential for an accurate description of magnetic moments. We observe that without the time-odd mean fields, the DFT value obtained for ${ }^{131}$ In is close to the single-particle value. Thus, the quadrupole deformation of this nucleus cannot explain the experimental value. However, the addition of a spin-spin parameter corresponding to the isovector Landau parameter ${ }^{40}$ of $g_{0}^{\prime}=0.82$ generates time-odd mean fields that result in a perfect agreement between the DFT and the experimental ${ }^{131}$ In magnetic moment. Similarly as for the $Q$ moments, we find that the HF-variant of the DFT better matches experiment around $N=82$, reproducing the magnitude of the jump in $\mu$ well in contrast to those including pairing correlations (HFB).

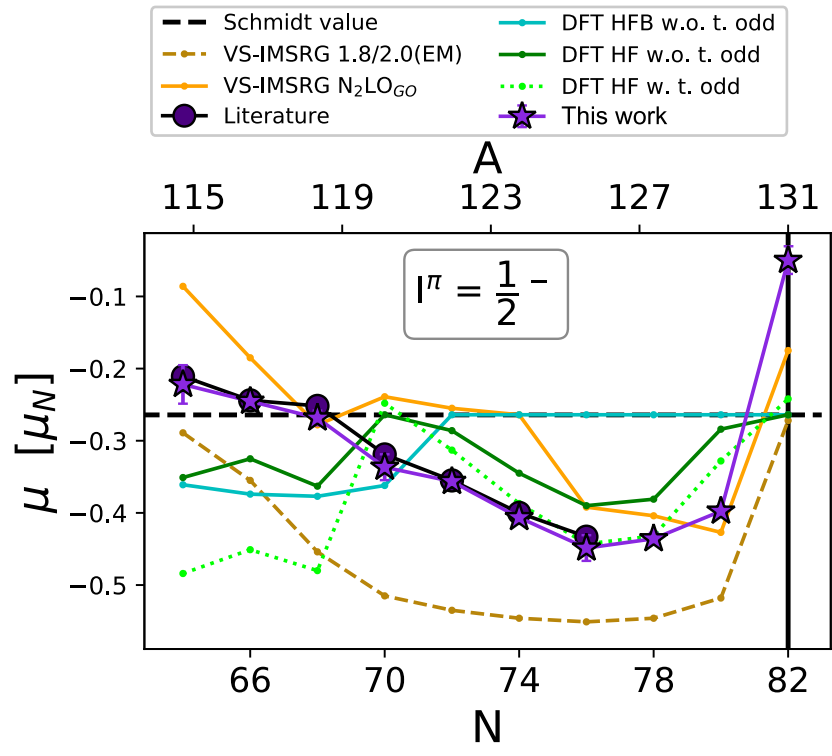

Figure 2. Nuclear magnetic moments for the $1 / 2^{-}$isomeric states of ${ }^{113-131}$ In isotopes. Results are compared with $a b$ initio and Density Functional Theory calculations. The horizontal dotted line indicates the single-particle value (Schmidt line). See text for more details

In order to further investigate the abrupt change that was observed at $N=82$ within the DFT framework, we traced back the properties of $\mu$ in indium directly to those of their deformed and polarised cores. To this end, we performed symmetry-restoration calculations by removing the deformed odd-proton orbitals from the self-consistent DFT states in indium or by filling in the corresponding odd-proton holes. In this way, we gained access to the corresponding $0^{+}, 1^{+}, 2^{+}$, $3^{+}, 4^{+}, \ldots$ states of the cadmium or tin cores. At this point, it is essential to recall that without the time-odd mean fields the core is not spin-polarised and therefore its states conserve signature symmetry and odd-angular-momentum components vanish. The effect of the time-odd mean fields on magnetic moments thus proceeds through the coupling to the $1^{+}, 3^{+}$, $\ldots, 9^{+}$states of the polarised core. The DFT calculations indicate that the change in the $9 / 2^{+}$moments can be attributed to the dominance of the effect of the spin-distribution at the $N=82$ shell closure and the charge distribution for $N<82$. Predictions for $N>82$ using these DFT calculations are shown in Methods (Figure 4), supporting the suggestion of a possible new magic number at $N=90^{41}$.

Previously, the decreasing $\mu$ values of the $1 / 2^{-}$isomeric states with increasing neutron number (see Figure 2) were not explained by nuclear theory. Our new experimental results for ${ }^{129,131}$ In show a sudden increase, which crosses the extreme single-particle value at $N=82$. The results are compared with literature values of ${ }^{115-127}$ In in Table 1 . We find that the DFT calculations without pairing correlations also provide a better description than those with pairing correlations (HFB). The introduction of time-odd fields improves the agreement with experiment. However, the pronounced increase at $N=82$ is not reproduced within this framework, with predictions close to the single-particle limit. This suggests the $1 / 2^{-}$isomers are highly collective in nature even at $N=82$. Our VS-IMSRG calculations provide some insight here. Overall, these calculations provide a good description of the previously unexplained trend observed for the magnetic moments of the $1 / 2^{-}$states. Analysis of the VS-IMSRG calculations indicates that the $1 / 2^{-}$state contains a significant component resulting from the coupling between the $9 / 2^{+}$single-particle configuration and the $5^{-}$(anti-aligned neutrons in the $h_{11 / 2}$ and $s_{1 / 2}$ orbitals) state of the core. This therefore suggests that further refinement of the present DFT calculations would be needed to explicitly include other core configurations e.g. negative-parity states, to accurately describe the $1 / 2^{-}$states.

\section{Summary and Outlook}

The indium isotopes have been considered a textbook example for the dominance of single-particle properties in heavy nuclei. Here, we show that their ground-state electromagnetic properties significantly differ at $N=82$ compared to $N<82$, despite the single unpaired proton ( $\pi g_{9 / 2}$ proton orbit) dominating the behaviour of this complex many-body system.

Our new experimental results for ${ }^{129,131}$ In show that upon reaching the neutron-rich magic number $N=82$, abrupt changes of their electromagnetic properties are observed, and only at the neutron magic number the single-particle structure is almost fully recovered. This challenges our previous un- 
derstanding of these isotopes, which were assumed to have a single-particle description by invoking the use of empirical effective operators.

We presented two complementary nuclear models to investigate how these seemingly simple structures emerge from the complex interactions among nucleons. Both DFT and $a b$ initio calculations provide a good description of the experimental trends. Within the DFT framework, the intricate isotopic dependence of the indium magnetic and electric ground-state moments turns out to require correct treatment of spin and shape polarization exerted by the proton-hole on the $Z=50$ magic core. The inclusion of time-odd mean fields was shown to be essential to reproduce the experimental findings.

Indium nuclei can coexist in long-lived exited states with $I^{\pi}=1 / 2^{-}$. The structure of these states was suggested to be dominated by a single unpaired proton in the $\pi p_{1 / 2}$ orbit. However, in contrast to the simple trends observed for the ground states, the magnetic moments of the $1 / 2^{-}$states exhibit a large variation with neutron number, previously considered a puzzle in the region ${ }^{12}$. These features and the new experimental observations are well described by our ab initio calculations. Our new measurements show that in addition to previous observations, the trend of their magnetic moments changes, with a sudden uptick at $N=82$.

Although our ab initio calculations provide a good description of the experimental trends, they fall short in reproducing the magnitude of the electromagnetic moments. This may be attributed to the lack of inclusion of many-body currents, e.g. meson-exchange currents, which are known to be essential to describe the electromagnetic properties of light nuclei ${ }^{42}$. Future theoretical developments would be needed to include many-body currents and clarify the role that they play in heavy nuclei. On the other hand, a theoretical description of the nuclear quadrupole moments requires the extensive inclusion of neglected many-body correlations. Thus, our new experimental results at $N=82$ provide critical data for future developments of both $a b$ initio and DFT theory.

Our experimental results for ${ }^{131}$ In allowed an investigation of the strength of the time-odd mean fields, which until now have been poorly constrained in DFT theories. Such time-odd channels are essential for a correct description of numerous nuclear properties, such as double beta-decay rates ${ }^{8,9}$, permanent electric dipole moment measurements ${ }^{10,11}$, and dark matter searches ${ }^{36}$. This provides strong motivation to extend experiments to other isotopes possessing single-hole (particle) with respect to suggested nuclear closed shells at extreme proton-to-neutron ratios, and to further pursue theoretical studies of these systems.

\section{References}

1. Th Schmidt. The electric quadrupole moment of the nucleus. Nature, 138(3488):404, 1936.

2. K. L. Jones, A. S. Adekola, D. W. Bardayan, J. C. Blackmon, K. Y. Chae, K. A. Chipps, J. A. Cizewski, L. Erikson, C. Harlin, R. Hatarik, R. Kapler, R. L. Kozub, J. F.
Liang, R. Livesay, Z. Ma, B. H. Moazen, C. D. Nesaraja, F. M. Nunes, S. D. Pain, N. P. Patterson, D. Shapira, J. F. Shriner, M. S. Smith, T. P. Swan, and J. S. Thomas. The magic nature of 132 Sn explored through the singleparticle states of $133 \mathrm{Sn}$. Nature, 465(7297):454-457, may 2010.

3. R. Taniuchi, C. Santamaria, P. Doornenbal, A. Obertelli, K. Yoneda, G. Authelet, H. Baba, D. Calvet, F. Château, A. Corsi, A. Delbart, J. M. Gheller, A. Gillibert, J. D. Holt, T. Isobe, V. Lapoux, M. Matsushita, J. Menéndez, S. Momiyama, T. Motobayashi, M. Niikura, F. Nowacki, K. Ogata, H. Otsu, T. Otsuka, C. Péron, S. Péru, A. Peyaud, E. C. Pollacco, A. Poves, J. Y. Roussé, H. Sakurai, A. Schwenk, Y. Shiga, J. Simonis, S. R. Stroberg, S. Takeuchi, Y. Tsunoda, T. Uesaka, H. Wang, F. Browne, L. X. Chung, Z. Dombradi, S. Franchoo, F. Giacoppo, A. Gottardo, K. Hadyńska-Klęk, Z. Korkulu, S. Koyama, Y. Kubota, J. Lee, M. Lettmann, C. Louchart, R. Lozeva, K. Matsui, T. Miyazaki, S. Nishimura, L. Olivier, S. Ota, Z. Patel, E. Şahin, C. Shand, P. A. Söderström, I. Stefan, D. Steppenbeck, T. Sumikama, D. Suzuki, Z. Vajta, V. Werner, J. Wu, and Z. Y. Xu. 78 $\mathrm{Ni}$ revealed as a doubly magic stronghold against nuclear deformation. Nature, 569(7754):53-58, may 2019.

4. T Togashi, Y Tsunoda, T Otsuka, N Shimizu, and M Honma. Novel Shape Evolution in Sn Isotopes from Magic Numbers 50 to 82. Physical Review Letters, 121(6):062501, aug 2018.

5. David Gareth Jenkins. Recent advances in nuclear physics through on-line isotope separation. Nature Physics, 10(12):909-913, dec 2014.

6. K Heyde. The Nuclear Shell Model. Springer Series in Nuclear and Particle Physics. Springer Berlin Heidelberg, Berlin, Heidelberg, 1990.

7. D. Rosiak, M. Seidlitz, P. Reiter, H. Naidja, Y. Tsunoda, T. Togashi, F. Nowacki, T. Otsuka, G. Colo, K. Arnswald, T. Berry, A. Blazhev, M. J. G. Borge, J. Cederkall, D. M. Cox, H. De Witte, L. P. Gaffney, C. Henrich, R. Hirsch, M. Huyse, A. Illana, K. Johnston, L. Kaya, Th. Kröll, M. L. Lozano Benito, J. Ojala, J. Pakarinen, M. Queiser, G. Rainovski, J. A. Rodriguez, B. Siebeck, E. Siesling, J. Snall, P. Van Duppen, A. Vogt, M. von Schmid, N. Warr, F. Wenander, and K. O. Zell. Enhanced Quadrupole and Octupole Strength in Doubly Magic Sn 132. Physical Review Letters, 121(25):252501, dec 2018.

8. Jonathan Engel and Javier Menéndez. Status and future of nuclear matrix elements for neutrinoless doublebeta decay: a review. Reports on Progress in Physics, 80(4):046301, mar 2017.

9. Michelle J. Dolinski, Alan W.P. Poon, and Werner Rodejohann. Neutrinoless double-beta decay: Status and prospects. Annual Review of Nuclear and Particle Science, 69(1):219-251, 2019. 
10. Jacek Dobaczewski, Jonathan Engel, Markus Kortelainen, and Pierre Becker. Correlating Schiff moments in the light actinides with octupole moments. Phys. Rev. Lett., 121:232501, Dec 2018.

11. T. E. Chupp, P. Fierlinger, M. J. Ramsey-Musolf, and J. T. Singh. Electric dipole moments of atoms, molecules, nuclei, and particles. Rev. Mod. Phys., 91:015001, Jan 2019.

12. J. Eberz, U. Dinger, G. Huber, H. Lochmann, R. Menges, R. Neugart, R. Kirchner, O. Klepper, T. Kühl, D. Marx, G. Ulm, and K. Wendt. Spins, moments and mean square charge radii of 104-127In determined by laser spectroscopy. Nuclear Physics A, 464(1):9-28, mar 1987.

13. P. Gysbers, G. Hagen, J. D. Holt, G. R. Jansen, T. D. Morris, P. Navrátil, T. Papenbrock, S. Quaglioni, A. Schwenk, S. R. Stroberg, and K. A. Wendt. Discrepancy between experimental and theoretical $\beta$-decay rates resolved from first principles. Nature Physics, 15(5):428-431, may 2019.

14. T. D. Morris, J. Simonis, S. R. Stroberg, C. Stumpf, G. Hagen, J. D. Holt, G. R. Jansen, T. Papenbrock, R. Roth, and A. Schwenk. Structure of the Lightest Tin Isotopes. Physical Review Letters, 120(15):152503, apr 2018.

15. Nicolas Schunck, editor. Energy Density Functional Methods for Atomic Nuclei. 2053-2563. IOP Publishing, 2019.

16. L. V. Rodríguez, D. L. Balabanski, M. L. Bissell, K. Blaum, B. Cheal, G. De Gregorio, J. Ekman, R. F. Garcia Ruiz, A. Gargano, G. Georgiev, W. Gins, C. Gorges, H. Heylen, A. Kanellakopoulos, S. Kaufmann, V. Lagaki, S. Lechner, B. Maaß, S. Malbrunot-Ettenauer, R. Neugart, G. Neyens, W. Nörtershäuser, S. Sailer, R. Sánchez, S. Schmidt, L. Wehner, C. Wraith, L. Xie, Z. Y. Xu, X. F. Yang, and D. T. Yordanov. Doubly-magic character of $132 \mathrm{Sn}$ studied via electromagnetic moments of $133 \mathrm{Sn}$. Physical Review C, 102(5):051301, nov 2020.

17. C. B. Hinke, M. Böhmer, P. Boutachkov, T. Faestermann, H. Geissel, J. Gerl, R. Gernhäuser, M. Górska, A. Gottardo, H. Grawe, J. L. Grebosz, R. Krücken, N. Kurz, Z. Liu, L. Maier, F. Nowacki, S. Pietri, Zs Podolyák, K. Sieja, K. Steiger, K. Straub, H. Weick, H. J. Wollersheim, P. J. Woods, N. Al-Dahan, N. Alkhomashi, A. Ataç, A. Blazhev, N. F. Braun, I. T. Čeliković, T. Davinson, I. Dillmann, C. Domingo-Pardo, P. C. Doornenbal, G. De France, G. F. Farrelly, F. Farinon, N. Goel, T. C. Habermann, R. Hoischen, R. Janik, M. Karny, A. Kaşkaş, I. M. Kojouharov, Th Kröll, Y. Litvinov, S. Myalski, F. Nebel, S. Nishimura, C. Nociforo, J. Nyberg, A. R. Parikh, A. Procházka, P. H. Regan, C. Rigollet, H. Schaffner, C. Scheidenberger, S. Schwertel, P. A. Söderström, S. J. Steer, A. Stolz, and P. Strmeň. Superallowed Gamow-Teller decay of the doubly magic nucleus 100Sn. Nature, 486(7403):341-345, jun 2012.
18. V. Manea, J. Karthein, D. Atanasov, M. Bender, K. Blaum, T. E. Cocolios, S. Eliseev, A. Herlert, J. D. Holt, W. J. Huang, Yu. A. Litvinov, D. Lunney, J. Menéndez, M. Mougeot, D. Neidherr, L. Schweikhard, A. Schwenk, J. Simonis, A. Welker, F. Wienholtz, and K. Zuber. First glimpse of the $\mathrm{N}=82$ shell closure below $\mathrm{Z}=50$ from masses of neutron-rich cadmium isotopes and isomers. Physical Review Letters, 124(9):092502, jan 2020.

19. Gerda Neyens. Nuclear magnetic and quadrupole moments for nuclear structure research on exotic nuclei. $R e$ ports on Progress in Physics, 66(4):633-689, apr 2003.

20. S. Ragnar Stroberg, Scott K. Bogner, Heiko Hergert, and Jason D. Holt. Nonempirical Interactions for the Nuclear Shell Model: An Update. Annual Review of Nuclear and Particle Science, 69(1), oct 2019.

21. K. Hebeler, S. K. Bogner, R. J. Furnstahl, A. Nogga, and A. Schwenk. Improved nuclear matter calculations from chiral low-momentum interactions. Physical Review $C$, 83(3), mar 2011.

22. J. Simonis, S. R. Stroberg, K. Hebeler, J. D. Holt, and A. Schwenk. Saturation with chiral interactions and consequences for finite nuclei. Physical Review C, 96(1), jul 2017.

23. R. P. de Groote, J. Moreno, J. Dobaczewski, I. Moore, M. Reponen, B. K. Sahoo, and C. Yuan. Measurement of an unusually large magnetic octupole moment in ${ }^{45} \mathrm{Sc}$ challenges state-of-the-art nuclear-structure theory. arXiv:2005.00414, 2020.

24. K. Bennaceur, J. Dobaczewski, T. Haverinen, and M. Kortelainen. Properties of spherical and deformed nuclei using regularized pseudopotentials in nuclear DFT. Journal of Physics G: Nuclear and Particle Physics, 47(10):23, oct 2020 .

25. J. Dobaczewski, W. Nazarewicz, T. R. Werner, J. F. Berger, C. R. Chinn, and J. Dechargé. Mean-field description of ground-state properties of drip-line nuclei: Pairing and continuum effects. Physical Review $\mathrm{C}$ - Nuclear Physics, 53(6):2809-2840, jun 1996.

26. J. Dobaczewski, H. Flocard, and J. Treiner. Hartree-FockBogolyubov description of nuclei near the neutron-drip line. Nuclear Physics, Section A, 422(1):103-139, jun 1984.

27. J.A. Sheikh, J. Dobaczewski, P. Ring, L.M. Robledo, and C. Yannouleas. Symmetry restoration in mean-field approaches. arxiv:1901.06992, 2019.

28. T.E. E. Cocolios, R.P. P. de Groote, J. Billowes, M.L. L. Bissell, I. Budinčević, T. Day Goodacre, G.J. J. FarooqSmith, V.N. N. Fedosseev, K.T. T. Flanagan, S. Franchoo, R.F. F. Garcia Ruiz, W. Gins, H. Heylen, T. Kron, R. Li, K.M. M. Lynch, B.A. A. Marsh, G. Neyens, R.E. E. Rossel, S. Rothe, A.J. J. Smith, H.H. H. Stroke, K.D.A. 
D A Wendt, S.G. G. Wilkins, and X. Yang. Highresolution laser spectroscopy with the Collinear Resonance Ionisation Spectroscopy (CRIS) experiment at CERN-ISOLDE. Nuclear Instruments and Methods in Physics Research Section B: Beam Interactions with Materials and Atoms, 376:284-287, 2016.

29. B K Sahoo, A R Vernon, R F Garcia Ruiz, C L Binnersley, J Billowes, M L Bissell, T E Cocolios, G J Farooq-Smith, K T Flanagan, W Gins, R P de Groote, Á Koszorús, G Neyens, K M Lynch, F Parnefjord-Gustafsson, C M Ricketts, K D A Wendt, S G Wilkins, and X F Yang. Analytic response relativistic coupled-cluster theory: the first application to indium isotope shifts. New Journal of Physics, 22(1):012001, jan 2020.

30. R. F. Garcia Ruiz, A. R. Vernon, C. L. Binnersley, B. K. Sahoo, M. Bissell, J. Billowes, T. E. Cocolios, W. Gins, R. P. de Groote, K. T. Flanagan, A. Koszorus, K. M. Lynch, G. Neyens, C. M. Ricketts, K. D.A. A. Wendt, S. G. Wilkins, and X. F. Yang. High-Precision Multiphoton Ionization of Accelerated Laser-Ablated Species. Physical Review X, 8(4):041005, oct 2018.

31. Evgeny Epelbaum, H.-W. Hammer, and Ulf-G. Meißner. Modern theory of nuclear forces. Reviews of Modern Physics, 81(4):1773-1825, 2009.

32. R. Machleidt and D. R. Entem. Chiral effective field theory and nuclear forces. Physics Reports, 503(1):1-75, jun 2011.

33. W. G. Jiang, A. Ekström, C. Forssén, G. Hagen, G. R. Jansen, and T. Papenbrock. Accurate bulk properties of nuclei from $\mathrm{A}=2$ to $\infty$ from potentials with $\Delta$ isobars. Physical Review C, 102(5):054301, nov 2020.

34. Y.M. Engel, D.M. Brink, K. Goeke, S.J. Krieger, and D. Vautherin. Time-dependent Hartree-Fock theory with Skyrme's interaction. Nuclear Physics A, 249(2):215 238, 1975.

35. E. Perlińska, S. G. Rohoziński, J. Dobaczewski, and W. Nazarewicz. Local density approximation for protonneutron pairing correlations: Formalism. Phys. Rev. C, 69:014316, Jan 2004.

36. J. Engel, S. Pittel, and P. Vogel. Nuclear Physics of Dark Matter Detection. International Journal of Modern Physics E, 1(1):1-37, January 1992.

37. J. Henderson et al. Testing microscopically derived descriptions of nuclear collectivity: Coulomb excitation of ${ }^{22}$ Mg. Phys. Lett. B, 782:468-473, 2018.

38. D. T. Yordanov, D. L. Balabanski, J. Bieroń, M. L. Bissell, K. Blaum, I. Budinčević, S. Fritzsche, N. Frömmgen, G. Georgiev, Ch. Geppert, M. Hammen, M. Kowalska, K. Kreim, A. Krieger, R. Neugart, W. Nörtershäuser, J. Papuga, and S. Schmidt. Spins, electromagnetic moments, and isomers of ${ }^{107-129} \mathrm{Cd}$. Phys. Rev. Lett., 110:192501, May 2013.
39. Th Schmidt. Über die magnetischen Momente der Atomkerne. Zeitschrift für Physik, 106(5-6):358-361, may 1937.

40. M. Bender, J. Dobaczewski, J. Engel, and W. Nazarewicz. Gamow-Teller strength and the spin-isospin coupling constants of the Skyrme energy functional. Phys. Rev. C, 65:054322, May 2002.

41. S. Sarkar and M. Saha Sarkar. New shell closure for neutron-rich Sn isotopes. Physical Review C - Nuclear Physics, 81(6):1-6, 2010.

42. S. Pastore, Steven C. Pieper, R. Schiavilla, and R. B. Wiringa. Quantum Monte Carlo calculations of electromagnetic moments and transitions in $A \leq 9$ nuclei with meson-exchange currents derived from chiral effective field theory. Phys. Rev. C, 87:035503, Mar 2013.

\section{Methods}

\section{The collinear resonance ionisation setup}

The indium isotopes were produced at CERN's on-line isotope separator facility, ISOLDE, by impinging $1.4-\mathrm{GeV}$ protons onto the proton-to-neutron converter of a thick uranium-carbide target ${ }^{8}$. The converter suppressed nearby caesium mass contamination ${ }^{9}$. The indium isotopes diffused through the target material and their ionisation was significantly enhanced by the use of the resonance laser ion source, RILIS ${ }^{10}$. The produced indium ions were then accelerated to $40 \mathrm{keV}$ and mass separated using the ISOLDE high-resolution mass separator before being cooled and bunched using a gas-filled linear Paul trap (ISCOOL) $)^{11,12}$. After a trapping time of up to $10 \mathrm{~ms}$, ion bunches with a temporal width of $2 \mu$ s, were then re-accelerated to 40034(1) $\mathrm{eV}$ and deflected into the CRIS beamline ${ }^{13,14}$. The indium ions were then neutralised with a sodium-filled charge-exchange cell with an efficiency of up to $60 \%$ and predicted relative atomic populations of $57 \%$ and $37 \%$ respectively for the $5 \mathrm{p}^{2} \mathrm{P}_{3 / 2}$ metastable state and $5 \mathrm{p}^{2} \mathrm{P}_{1 / 2}$ ground state ${ }^{15}$. The remaining ion fraction was removed by electrostatic deflectors, and the neutralized atom bunch was collinearly overlapped with two pulsed lasers, one for excitation and another for non-resonant ionisation. The atoms were resonantly excited using two different $\mathrm{UV}$ transitions in separate measurements. The first using 246.8-nm laser light for the $5 \mathrm{p}^{2} \mathrm{P}_{3 / 2} \rightarrow 9 \mathrm{~s}{ }^{2} \mathrm{~S}_{1 / 2}$ atomic transition. The second using 246.0-nm laser light for the $5 \mathrm{p}^{2} \mathrm{P}_{1 / 2} \rightarrow 8 \mathrm{~s}^{2} \mathrm{~S}_{1 / 2}$ atomic transition, which is more sensitive to nuclear magnetic moments, $\mu$, but alone does not give the nuclear electric quadrupole moment, $Q$. The resonant laser light was produced by frequency tripling the light from an injection-locked Ti:Sapphire laser system ${ }^{16}$. This laser was seeded using a narrowband SolsTiS continuous-wave Ti:Sapphire laser, and pumped using a Lee Laser LDP-100MQ Nd:YAG laser, producing pulsed narrowband 740(738)-nm laser light at $1 \mathrm{kHz}$ repetition rate. This light was then frequency tripled to $246.8(246.0)$-nm light by the use of two non-linear crystals ${ }^{17}$. About $3 \mu \mathrm{J}$ of laser light was used to saturate both transitions. The excited atoms were then ionized by a final nonresonant 1064-nm step, provided by a Litron LPY 601 50-100 PIV $\mathrm{Nd}: Y A G$ laser at $100 \mathrm{~Hz}$ repetition rate. The frequency of the resonant first step was scanned and the resulting ions were deflected onto a detector, producing the hyperfine structure containing spectra from which the hyperfine parameters were obtained. The wavelengths were measured using a HighFinesse WSU-2 wavemeter, which was 

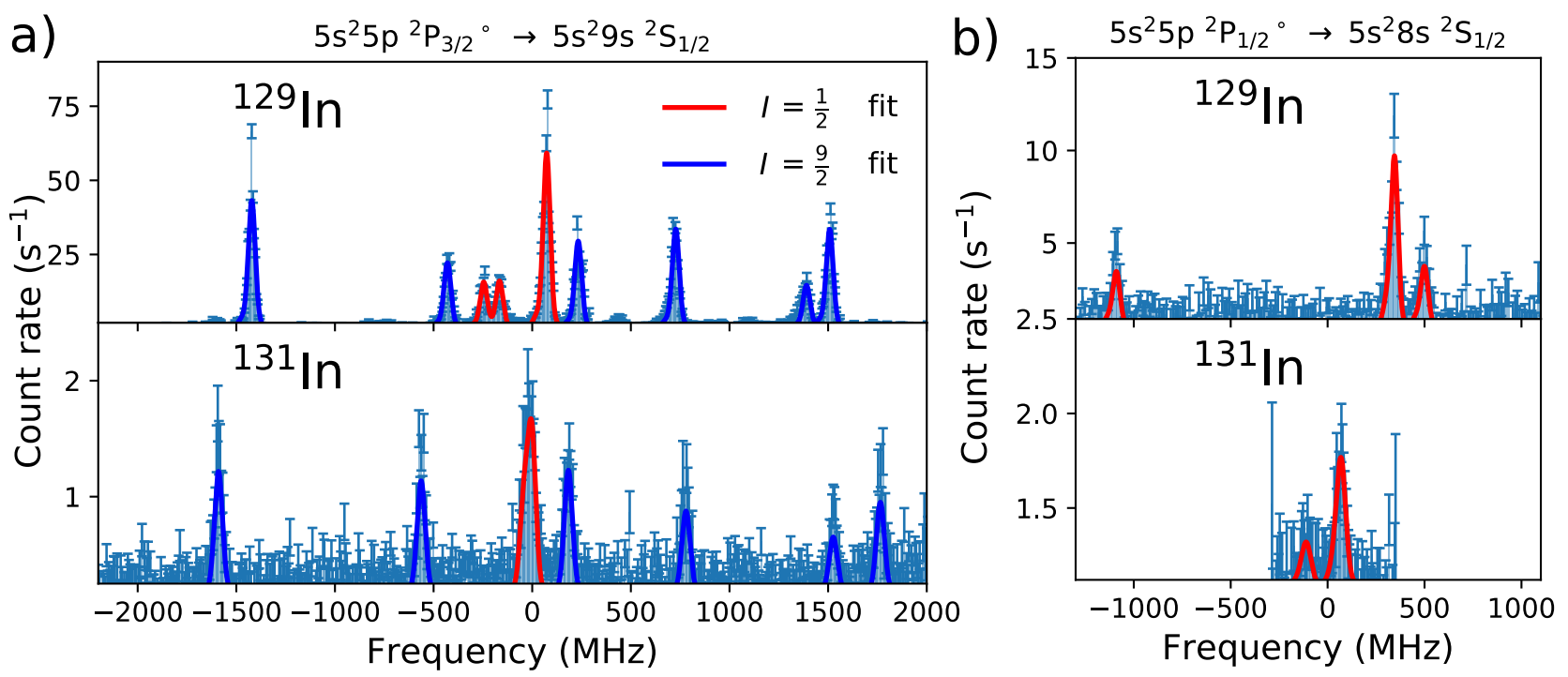

Figure 3. (color online) a) Example hyperfine spectra of the ${ }^{129} \mathrm{In}$ and ${ }^{131} \mathrm{In}$ isotopes, measured using the $246.8-\mathrm{nm}\left(5 \mathrm{p}{ }^{2} \mathrm{P}_{3 / 2}\right.$ $\rightarrow 9 \mathrm{~s}^{2} \mathrm{~S}_{1 / 2}$ ) transition. The $9 / 2^{+}$ground and $1 / 2^{-}$isomer states are indicated. b) Example spectra of the $1 / 2^{-}$isomer structure measured with the $246.0-\mathrm{nm}\left(5 \mathrm{p}^{2} \mathrm{P}_{1 / 2} \rightarrow 8 \mathrm{~s}^{2} \mathrm{~S}_{1 / 2}\right)$ transition.

Table 1. The magnetic hyperfine structure parameters, $A_{\mathrm{hf}}$, measured in this work for the odd-mass ${ }^{113-131}$ In isotopes and corresponding extracted magnetic dipole moment values.

\begin{tabular}{|c|c|c|c|c|c|c|c|}
\hline \multirow[b]{2}{*}{ A } & \multirow[b]{2}{*}{ I } & \multicolumn{5}{|c|}{$\mathrm{A}_{h f}(\mathrm{MHz})$} & \multirow[b]{2}{*}{$\mu^{\text {Lit. }}\left(\mu_{N}\right)$} \\
\hline & & $5 p^{2} \mathrm{P}_{3 / 2}$ & $9 \mathrm{~s}^{2} \mathrm{~S}_{1 / 2}$ & $5 p^{2} P_{1 / 2}$ & $8 s^{2} S_{1 / 2}$ & $\mu^{\ddagger}\left(\mu_{N}\right)$ & \\
\hline 105 & $9 / 2^{+}$ & & & & & & $+5.675(5)^{1}$ \\
\hline 107 & $9 / 2^{+}$ & & & & & & $+5.585(8)^{1}$ \\
\hline 109 & $9 / 2^{+}$ & & & & & & $+5.538(4)^{1}$ \\
\hline 111 & $9 / 2^{+}$ & & & & & & $+5.503(7)^{1}$ \\
\hline 113 & $9 / 2^{+}$ & $+241.8(8)$ & $+130(1)$ & $+2276.0(8)$ & $+242.7(8)$ & $+5.5264(19)$ & $+5.5289(2)^{2}$ \\
\hline 113 & $1 / 2^{-}$ & $-87(10)$ & $-38(20)$ & $-774(50)$ & $-90(50)$ & $-0.21(1)$ & $-0.21074(2)^{3}$ \\
\hline 115 & $9 / 2^{+}$ & $+239.9(5)$ & $+130.3(8)$ & $+2281.9(8)$ & $+243.3(6)$ & $+5.5408(2)^{\ddagger}$ & $+5.5408(2)^{4}$ \\
\hline 115 & $1 / 2^{-}$ & $-96(3)$ & $-48(9)$ & $-903(20)$ & $-66(10)$ & $-0.2405(38)$ & $-0.24398(5)^{5}$ \\
\hline 117 & $9 / 2^{+}$ & $+241(4)$ & $+130(5)$ & $+2277(2)$ & $+244(2)$ & $+5.5286(43)$ & $+5.519(4)^{1}$ \\
\hline 117 & $1 / 2^{-}$ & $-106(4)$ & $-49(10)$ & $-1028(10)$ & $-113(8)$ & $-0.276(27)$ & $-0.25174(3)^{1}$ \\
\hline 119 & $9 / 2^{+}$ & $+240(4)$ & $+130(5)$ & & & $+5.499(62)$ & $+5.515(1)^{1}$ \\
\hline 119 & $1 / 2^{-}$ & $-132(7)$ & $-70(10)$ & & & $-0.342(12)$ & $-0.319(5)^{1}$ \\
\hline 121 & $9 / 2^{+}$ & $+243(4)$ & $133(5)$ & & & $+5.575(62)$ & $+5.502(5)^{1}$ \\
\hline 121 & $1 / 2^{-}$ & $-140(2)$ & $-85(6)$ & & & $-0.3600(41)$ & $-0.355(4)^{1}$ \\
\hline 123 & $9 / 2^{+}$ & $+238(4)$ & $+129(5)$ & & & $+5.442(61)$ & $+5.491(7)^{1}$ \\
\hline 123 & $1 / 2^{(-)}$ & $-160(2)$ & $-80(5)$ & & & $-0.4047(54)$ & $-0.400(4)^{1}$ \\
\hline 125 & $9 / 2^{+}$ & $+240.3(6)$ & $+129.9(8)$ & & & $+5.496(24)$ & $+5.502(9)^{1}$ \\
\hline 125 & $1 / 2^{(-)}$ & $-176(7)$ & $-90(10)$ & & & $-0.450(17)$ & $-0.433(4)^{1}$ \\
\hline 127 & $9 / 2^{+}$ & $+241.8(7)$ & $+130(1)$ & $+2278.3(6)$ & $+243.8(4)$ & $+5.5321(14)$ & $+5.522(8)^{1}$ \\
\hline 127 & $1 / 2^{(-)}$ & $-171(3)$ & $-91(10)$ & $-1613(9)$ & $-174(8)$ & $-0.4355(24)$ & \\
\hline 129 & $\left(9 / 2^{+}\right)$ & $+243.3(8)$ & $+132(1)$ & $+2304.9(9)$ & $+244.8(7)$ & $+5.5961(23)$ & \\
\hline 129 & $1 / 2^{(-)}$ & $-156(3)$ & $-80(4)$ & $-1434(2)$ & $-162(10)$ & $-0.3871(6)$ & \\
\hline 131 & $\left(9 / 2^{+}\right)$ & $+275.9(6)$ & $+149.3(7)$ & & & $+6.312(14)$ & \\
\hline 131 & $1 / 2^{(-)}$ & $-20(7)$ & $-11(4)$ & $-188(20)$ & $-20(2)$ & $-0.051(3)$ & \\
\hline
\end{tabular}


Table 2. The $B_{\text {hf }}$ hyperfine structure parameter (from the ${ }^{2} \mathrm{P}_{3 / 2}$ state) values, measured in this work for the odd-mass ${ }^{113-131}$ In isotopes and the extracted electric quadrupole moment values.

\begin{tabular}{|c|c|c|c|c|}
\hline A & I & $\mathrm{B}_{h f}(\mathrm{MHz})$ & $\mathrm{Q}_{S}^{\dagger}(\mathrm{b})$ & $\mathrm{Q}_{S}^{\text {Lit. (b) }}$ \\
\hline 105 & $9 / 2^{+}$ & & & $+0.79(5)^{1,6}$ \\
\hline 107 & $9 / 2^{+}$ & & & $+0.77(5)^{1,6}$ \\
\hline 109 & $9 / 2^{+}$ & & & $+0.80(3)^{1,6}$ \\
\hline 111 & $9 / 2^{+}$ & & & $+0.76(2)^{1,6}$ \\
\hline 113 & $9 / 2^{+}$ & $+441(15)$ & $+0.767(27)$ & $+0.759(8)^{1,6}$ \\
\hline 115 & $9 / 2^{+}$ & $+454.2(65)$ & $+0.789(13)$ & $+0.770(8)^{1,6}$ \\
\hline 117 & $9 / 2^{+}$ & $+465(13)$ & $+0.807(23)$ & $+0.788(10)^{1,6}$ \\
\hline 119 & $9 / 2^{+}$ & $+462(13)$ & $+0.802(23)$ & $+0.812(7)^{1,6}$ \\
\hline 121 & $9 / 2^{+}$ & $+462(13)$ & $+0.803(23)$ & $+0.774(10)^{1,6}$ \\
\hline 123 & $9 / 2^{+}$ & $+424(13)$ & $+0.736(23)$ & $+0.720(9)^{1,6}$ \\
\hline 125 & $9 / 2^{+}$ & $+382.3(55)$ & $+0.664(11)$ & $+0.68(3)^{1,6}$ \\
\hline 127 & $9 / 2^{+}$ & $+338(16)$ & $+0.588(29)$ & $+0.56(3)^{1,6}$ \\
\hline 129 & $\left(9 / 2^{+}\right)$ & $+280.4(73)$ & $+0.487(13)$ & \\
\hline 131 & $\left(9 / 2^{+}\right)$ & $+177.3(57)$ & $+0.310(10)$ & \\
\hline
\end{tabular}

drift stabilized by simultaneous measurement of a Toptica DLC DL PRO 780 diode laser locked to the $5 \mathrm{~s}^{2} \mathrm{~S}_{1 / 2} \rightarrow 5 \mathrm{p}^{2} \mathrm{P}_{3 / 2} \mathrm{~F}=2 \rightarrow 3$ transition of ${ }^{87} \mathrm{Rb}$ using a saturated absorption spectroscopy unit.

\section{Evaluation of nuclear magnetic and quadrupole moments}

The $\mu$ values were determined using a reference NMR value of $\mu_{\text {ref }}=+5.5408(2) \mu_{N}$ for ${ }^{115} \mathrm{In}^{4}$, and the relation

$$
\mu=\mu_{r e f} \frac{I A}{I_{r e f}, A_{r e f}}(1+\Delta),
$$

where the differential hyperfine anomaly, $\Delta$, is negligible for these atomic states of indium ${ }^{18}$. Here $A_{\text {ref }}$ are our experimentally determined values for the $5 \mathrm{p}^{2} \mathrm{P}_{3 / 2}, 9 \mathrm{~s}^{2} \mathrm{~S}_{1 / 2}, 5 \mathrm{p}^{2} \mathrm{P}_{1 / 2}$ and $8 \mathrm{~s}^{2} \mathrm{~S}_{1 / 2}$ states of stable ${ }^{115} \mathrm{In}$, and $A$ are those of short-lived isotopes. The final $\mu$ values presented in Table 1 are a weighted average of the $\mu$ values from each atomic state, which were self-consistent within $2 \sigma$.

The spectroscopic nuclear electric quadrupole moments, $Q$, were extracted using the relation

$$
B_{h f}=e Q V_{Z Z},
$$

where a value of $B_{h f}\left({ }^{2} \mathrm{P}_{3 / 2}\right) / Q=576(4) \mathrm{MHz} / \mathrm{b}$ was used, obtained from relativistic coupled-cluster atomic calculations ${ }^{7}$. Here $V_{Z Z}$ is the electric field gradient produced by the electrons at the nucleus.

The ratio of $A_{h f}$ factors is depends dominantly on atomic structure and therefore remains constant when with the correct nuclear spin assignment is used for fitting of spectra resulting from hyperfine structure $^{19}$. Although reduced $\chi^{2}$ fitting of the spectra (as shown in Figure 3) resulted in minima when we use $I=9 / 2$; the spin assignment from ratio of upper and lower atomic state $A_{h f}$ factors (of the $5 \mathrm{p}^{2} \mathrm{P}_{3 / 2} \rightarrow 9 \mathrm{~s}{ }^{2} \mathrm{~S}_{1 / 2}$ transition) could not rule out nuclear spins $7 / 2,11 / 2$, outside of $1 \sigma$ uncertainty. The spin of the $I=9 / 2$ states are tentatively assigned by experiment and strongly supported by nuclear theory. The spin assignments of the $I=1 / 2$ states were confirmed unambiguously from the number of peaks in their spectra.

\section{VS-IMSRG calculations}

The VS-IMSRG calculations start from the underlying NN and $3 \mathrm{~N}$ interactions expressed in the harmonic-oscillator basis. We then construct an effective Hamiltonian designed for a particular valence space where the exact diagonalization is feasible ${ }^{20}$. The effective Hamiltonian was decoupled from the full $A$-body Hamiltonian through the application of an approximate unitary transformation derived from the Magnus expansion method ${ }^{20}$. Using the same transformation, the effective $M 1$ and $E 2$ valence-space operators were then decoupled consistently with the Hamiltonian. During the calculation, all of operators arising in nested commutator expansions were truncated at the two-body level, the IMSRG(2) approximation.

We begin in a spherical harmonic-oscillator basis with frequency of $\hbar \omega=16 \mathrm{MeV}$ and $e=2 n+l \leq e_{\max }=14$ for 1.8/2.0 (EM) (and 12 for $\mathrm{N}^{2} \mathrm{LO}_{\mathrm{GO}}$ ) with an additional cut of $e_{1}+e_{2}+e_{3} \leq E_{3 \max }=16$ imposed due to storage limitations of $3 \mathrm{~N}$ matrix elements. The valence space was taken as the proton $\left\{1 p_{1 / 2}, 1 p_{3 / 2}, 0 f_{5 / 2}, 0 g_{9 / 2}\right\}$ and neutron $\left\{2 s_{1 / 2}, 1 d_{3 / 2}, 1 d_{5 / 2}, 0 g_{7 / 2}, 0 h_{11 / 2}\right\}$ single-particle orbits above ${ }^{78} \mathrm{Ni}$ core. The final valence-space diagonalization and evaluation of electromagnetic moments were performed with the KSHELL code ${ }^{21}$. The effect of fully accounting for translation invariance in the $M 1$ operator was found to be less than $1 \%$, largely due to the lack of radial dependence of the $M 1$ operator.

\section{DFT calculations}

We performed the DFT calculations using code HFODD ${ }^{22,23}$ version $(3.01 \mathrm{~m})$ and a standard Skyrme density functional UNEDF $1^{24}$. The deformed DFT single-particle wave functions were expanded on the spherical harmonic-oscillator basis up to $N_{0}=16$ quanta. An $A$-dependent harmonic-oscillator frequency $\hbar \omega$ was fixed according to the prescription defined in Ref. ${ }^{25}$. For the state $9 / 2^{+}$, the proton configuration (with no pairing correlations) was fixed by occupying 49 lowest axially deformed prolate orbitals, that is, the Nilsson orbital $^{26}[404] \frac{9}{2}$ with the angular-momentum projection on the symmetry axis $\Omega=-9 / 2$ was left empty. Similarly, for the state $1 / 2^{-}$, the hole was kept in the oblate Nilsson orbital [301] $\frac{1}{2}$ with $\Omega=-1 / 2$. It was essential to pick the proton-hole configurations with angular 


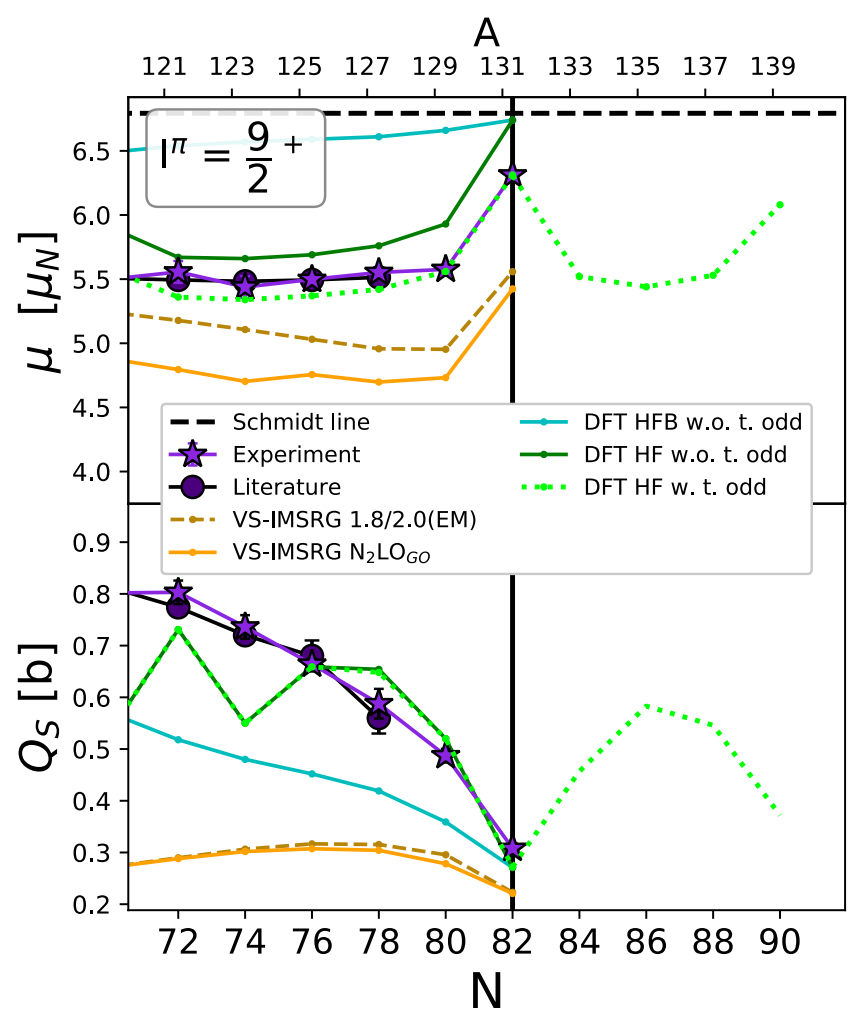

Figure 4. (color online) The a) nuclear magnetic dipole moments and b) electric quadrupole moments, respectively, of the $9 / 2^{+}$states extracted by our experiment. Shown alongside our $a b$ initio and DFT calculations, including predictions for $N>82$ momenta properly aligned along the axial-symmetry axis. Indeed, as discussed in Refs. ${ }^{27,28}$, the time-odd mean fields and core spin polarization depend on the relative orientation of the intrinsic angular momentum and shape.

For both proton configurations, the open-shell neutron configurations were constructed either with (HFB results) or without (HF results) pairing correlations included. For all deformed mean-field configurations, the angular-momentum symmetry was restored ${ }^{27}$, and in addition, for paired configurations the particle-number symmetry was restored as well ${ }^{27}$. Neither effective charges nor effective $g$-factors were used in the $a b$ initio or DFT calculations.

Figure 4 extends Figure 1, showing the DFT predictions of the magnetic dipole and electric quadrupole moments in indium, in an as-yet experimentally inaccessible region beyond the $N=82$ shell closure. The confirmation of the moments at $N=90$ would give strong evidence to support the existence of a new magic number of $N=90$ in this neutron-rich tin region, for which theoretical and experimental evidence is beginning to hint towards ${ }^{29-31}$.

\section{References}

1. J. Eberz, U. Dinger, G. Huber, H. Lochmann, R. Menges, R. Neugart, R. Kirchner, O. Klepper, T. Kühl, D. Marx, G. Ulm, and $\mathrm{K}$. Wendt. Spins, moments and mean square charge radii of 104-127In determined by laser spectroscopy. Nuclear Physics A, 464(1):9-28, mar 1987.

2. Michael Rice and R. V. Pound. Ratio of the Magnetic Moments of In 115 and In 113. Physical Review, 106(5):953-953, jun 1957.

3. W. J. Childs and L. S. Goodman. Nuclear Spin and Hyperfine Interaction of In 113m. Physical Review, 118(6):1578-1581, jun 1960.

4. C P Flynn and E F W Seymour. Knight Shift of the Nuclear Magnetic Resonance in Liquid Indium. Proceedings of the Physical Society, 76(2):301-303, aug 1960.

5. J. A. Cameron, H. J. King, H. K. Eastwood, and R. G. SummersGill. THE MAGNETIC MOMENT OF INDIUM-115m. Canadian Journal of Physics, 40(8):931-942, aug 1962.

6. N. J. Stone. Nuclear Magnetic Dipole and Electric Quadrupole Moments: Their Measurement and Tabulation as Accessible Data. Journal of Physical and Chemical Reference Data, 44(3):031215, sep 2015.

7. R. F. Garcia Ruiz, A. R. Vernon, C. L. Binnersley, B. K. Sahoo, M. Bissell, J. Billowes, T. E. Cocolios, W. Gins, R. P. de Groote, K. T. Flanagan, A. Koszorus, K. M. Lynch, G. Neyens, C. M. Ricketts, K. D.A. A. Wendt, S. G. Wilkins, and X. F. Yang. HighPrecision Multiphoton Ionization of Accelerated Laser-Ablated Species. Physical Review X, 8(4):041005, oct 2018.

8. U. Köster. Intense radioactive-ion beams produced with the ISOL method. The European Physical Journal A, 15(1-2):255263, sep 2002.

9. I. Dillmann, M. Hannawald, U. Köster, V.N. Fedoseyev, A. Wöhr, B. Pfeiffer, D. Fedorov, J. Shergur, L. Weissman, W.B. Walters, and K.-L. Kratz. Selective laser ionization of $\mathrm{N}>=82$ indium isotopes: The new r-process nuclide 135In. The European Physical Journal A, 13(3):281-284, mar 2002.

10. S Rothe, B A Marsh, C Mattolat, V N Fedosseev, and K Wendt. A complementary laser system for ISOLDE RILIS. Journal of Physics: Conference Series, 312(5):052020, sep 2011. 
11. E. Mané, J. Billowes, K. Blaum, P. Campbell, B. Cheal, P. Delahaye, K. T. Flanagan, D. H. Forest, H. Franberg, C. Geppert, T. Giles, A. Jokinen, M. Kowalska, R. Neugart, G. Neyens, W. Nörtershäuser, I. Podadera, G. Tungate, P. Vingerhoets, and D. T. Yordanov. An ion cooler-buncher for high-sensitivity collinear laser spectroscopy at ISOLDE. European Physical Journal A, 42(3):503-507, 2009.

12. H. Frånberg, P. Delahaye, J. Billowes, K. Blaum, R. Catherall, F. Duval, O. Gianfrancesco, T. Giles, A. Jokinen, M. Lindroos, D. Lunney, E. Mane, and I. Podadera. Off-line commissioning of the ISOLDE cooler. Nuclear Inst. and Methods in Physics Research, B, 266(19-20):4502-4504, 2008.

13. A. R. Vernon, R. P. de Groote, J. Billowes, C. L. Binnersley, T. E. Cocolios, G. J. Farooq-Smith, K. T. Flanagan, R. F. Garcia Ruiz, W. Gins, Koszorús, G. Neyens, C. M. Ricketts, A. J. Smith, S. G. Wilkins, and X. F. Yang. Optimising the Collinear Resonance Ionisation Spectroscopy (CRIS) experiment at CERN-ISOLDE. Nuclear Instruments and Methods in Physics Research, Section B: Beam Interactions with Materials and Atoms, jan 2019.

14. T.E. Cocolios, R.P. de Groote, J. Billowes, M.L. Bissell, I. Budinčević, T. Day Goodacre, G.J. Farooq-Smith, V.N. Fedosseev, K.T. Flanagan, S. Franchoo, R.F. Garcia Ruiz, W. Gins, H. Heylen, T. Kron, R. Li, K.M. Lynch, B.A. Marsh, G. Neyens, R.E. Rossel, S. Rothe, A.J. Smith, H.H. Stroke, K.D.A. Wendt, S.G. Wilkins, and X. Yang. High-resolution laser spectroscopy with the Collinear Resonance Ionisation Spectroscopy (CRIS) experiment at CERN-ISOLDE. Nuclear Instruments and Methods in Physics Research Section B: Beam Interactions with Materials and Atoms, 376:284-287, 2016.

15. A.R. Vernon, J. Billowes, C.L. L. Binnersley, M.L. L. Bissell, T.E. E. Cocolios, G.J. J. Farooq-Smith, K.T. T. Flanagan, R.F. F. Garcia Ruiz, W. Gins, R.P. P. de Groote, Á. Koszorús, K.M. M. Lynch, G. Neyens, C.M. M. Ricketts, K.D.A. D.A. Wendt, S.G. G. Wilkins, X.F. F. Yang, Koszorús, K.M. M. Lynch, G. Neyens, C.M. M. Ricketts, K.D.A. D.A. Wendt, S.G. G. Wilkins, and X.F. F. Yang. Simulation of the relative atomic populations of elements $1<=Z<=89$ following charge exchange tested with collinear resonance ionization spectroscopy of indium. Spectrochimica Acta Part B: Atomic Spectroscopy, 153:61-83, mar 2019.

16. V Sonnenschein, I D Moore, S Raeder, M Reponen, H Tomita, and $\mathrm{K}$ Wendt. Characterization of a pulsed injection-locked Ti:sapphire laser and its application to high resolution resonance ionization spectroscopy of copper. Laser Physics, 27(8):085701, aug 2017.

17. M. Bass, P. A. Franken, A. E. Hill, C. W. Peters, and G. Weinreich. Optical Mixing. Physical Review Letters, 8(1):18-18, jan 1962.

18. J.R. R. Persson. Table of hyperfine anomaly in atomic systems. Atomic Data and Nuclear Data Tables, 99(1):62-68, jan 2013.

19. B. Cheal, E. Mané, J. Billowes, M. L. Bissell, K. Blaum, B. A. Brown, F. C. Charlwood, K. T. Flanagan, D. H. Forest, C. Geppert, M. Honma, A. Jokinen, M. Kowalska, A. Krieger, J. Krämer, I. D. Moore, R. Neugart, G. Neyens, W. Nörtershäuser, M. Schug, H. H. Stroke, P. Vingerhoets, D. T. Yordanov, and M. Žáková. Nuclear spins and moments of Ga isotopes reveal sudden structural changes between $\mathrm{N}=40$ and $\mathrm{N}=50$. Physical Review Letters, 104(25):252502, jun 2010.
20. T D Morris, N M Parzuchowski, and S K Bogner. Magnus expansion and in-medium similarity renormalization group. Phys. Rev. C, 92(3):34331, sep 2015.

21. Noritaka Shimizu, Takahiro Mizusaki, Yutaka Utsuno, and Yusuke Tsunoda. Thick-restart block Lanczos method for largescale shell-model calculations. Comput. Phys. Commun., jun 2019.

22. N. Schunck, J. Dobaczewski, W. Satuła, P. Bączyk, J. Dudek, Y. Gao, M. Konieczka, K. Sato, Y. Shi, X.B. Wang, and T.R. Werner. Solution of the Skyrme-Hartree-Fock-Bogolyubov equations in the Cartesian deformed harmonic-oscillator basis. (VIII) HFODD (v2.73y): A new version of the program. Comp. Phys. Commun., 216:145 - 174, 2017.

23. J. Dobaczewski, P. Ba̧czyk, P. Becker, M. Bender, K. Bennaceur, J. Bonnard, Y. Gao, A. Idini, M. Konieczka, M. Kortelainen, L. Próchniak, A. M. Romero, W. Satuła, Y. Shi, T. R. Werner, and L. F. Yu. Solution of universal nonrelativistic nuclear DFT equations in the Cartesian deformed harmonic-oscillator basis. (IX) HFODD (v3.04n): a new version of the program. arXiv:2104.08255, apr 2021.

24. M. Kortelainen, J. McDonnell, W. Nazarewicz, P.-G. Reinhard, J. Sarich, N. Schunck, M. V. Stoitsov, and S. M. Wild. Nuclear energy density optimization: Large deformations. Phys. Rev. C, 85:024304, Feb 2012.

25. J. Dobaczewski and J. Dudek. Solution of the Skyrme-HartreeFock equations in the Cartesian deformed harmonic oscillator basis II. The program HFODD. Comput. Phys. Comm., 102(1):183 $-209,1997$.

26. K. Heyde, M. Waroquier, H. Vincx, and P. Van Isacker. Coexistence of spherical and deformed states near closed shells. Physics Letters B, 64(2):135-139, sep 1976.

27. N. Schunck, J. Dobaczewski, J. McDonnell, J. Moré, W. Nazarewicz, J. Sarich, and M. V. Stoitsov. One-quasiparticle states in the nuclear energy density functional theory. Phys. Rev. $C, 81: 024316$, Feb 2010.

28. W. Satuła, P. Bączyk, J. Dobaczewski, and M. Konieczka. No-core configuration-interaction model for the isospin- and angular-momentum-projected states. Phys. Rev. C, 94:024306, Aug 2016.

29. J. Meng and P. Ring. Giant halo at the neutron drip line. Physical Review Letters, 80(3):460-463, 1998.

30. S. Sarkar and M. Saha Sarkar. New shell closure for neutron-rich Sn isotopes. Physical Review C - Nuclear Physics, 81(6):1-6, 2010.

31. Z. P. Li, T. Nikšić, D. Vretenar, J. Meng, G. A. Lalazissis, and P. Ring. Microscopic analysis of nuclear quantum phase transitions in the $\mathrm{N} 90$ region. Physical Review $C$ - Nuclear Physics, 79(5):1-14, 2009.

\section{Acknowledgements}

This work was supported by ERC Consolidator Grant No.648381 (FNPMLS); STFC grants ST/L005794/1, ST/L005786/1, ST/P004423/1, ST/M006433/1, ST/P003885/1 and Ernest Rutherford Grant No. ST/L002868/1;the U.S. Department of Energy, Office of Science, Office of Nuclear Physics under grant DESC0021176; GOA 15/010 from KU Leuven, BriX Research 
Program No. P7/12; the FWO-Vlaanderen (Belgium); the European Unions Grant Agreement 654002 (ENSAR2); National Key R\&D Program of China (Contract No: 2018YFA0404403); the National Natural Science Foundation of China (No:11875073); the Polish National Science Centre under Contract No. 2018/31/B/ST2/02220. TRIUMF receives funding via a contribution through the National Research Council of Canada. The theoretical work was further supported by NSERC and the US Department of Energy (DOE) under contract DE-FG02-97ER41014. The VS-IMSRG Computations were performed with an allocation of computing resources on Cedar at WestGrid and Compute Canada, and on the Oak Cluster at TRIUMF managed by the University of British Columbia department of Advanced Research Computing (ARC). We would also like to thank the ISOLDE technical group for their support and assistance and the University of Jyväskylä for the use of the injection-locked cavity. We acknowledge the CSC-IT Center for Science Ltd., Finland, for the allocation of computational resources. This project was partly undertaken on the Viking Cluster, which is a high performance compute facility provided by the University of York. We are grateful for computational support from the University of York High Performance Computing service, Viking and the Research Computing team.

\section{Author contributions statement}

A.R.V. prepared the manuscript with input from all authors, especially R.F.G.R., J.Bo., J.D., J.D.H., T.M., G.N., K.T.F., T.E.C., R.P.G. and S.R.S. .

R.F.G., J.B., C.L.B., M.L.B., T.E.C., K.T.F., W.G., R.P.G., A.K., K.M.L., G.N., S.G.W., A.R.V. and X.F.Y. proposed the experiment(s),

A.R.V., C.L.B., M.L.B., T.E.C., K.T.F., G.J.F., G.G., W.G., R.P.G., R.H., A.K., D.L., K.M.L., R.F.G., S.G.W., X.F.Y. and D.Y. conducted the experiment(s),

A.R.V. and C.L.B. analysed the results,

J.Bo. and J.D. performed theoretical (DFT) nuclear calculations,

J.D.H., T.M., S.R.S. performed theoretical (VS-IMSRG) nuclear calculations.

All authors reviewed the manuscript. 